\title{
Abundances from a large spectroscopic survey in $\omega$ Centauri
}

\author{
A. Kayser ${ }^{1}$, M. Hilker ${ }^{2}$, T. Richtler ${ }^{3}$ and P. Willemsen ${ }^{2}$
}

${ }^{1}$ Astronomisches Institut der Universität Basel, Venusstrasse 7, 4102 Binningen, Switzerland

${ }^{2}$ Sternwarte der Universität Bonn, Auf dem Hügel 71, 53121 Bonn, Germany

${ }^{3}$ Universidad de Concepción, Departamento de Física, Casilla 106-C, Concepción, Chile

$\omega$ Centauri, the largest globular cluster of our Milky Way, is an outstanding object in many aspects. Studies on the red giant branch (RGB) revealed a large spread in iron abundance, which ranges from $[\mathrm{Fe} / \mathrm{H}] \sim-2.0$ to -0.4 dex and the existence of multiple sub-populations that do not only differ in their element abundances but also in their spatial and kinematic distributions (e.g. Norris et al. 1997). Furthermore more recent photometric studies suggested the existence of an age spread between these subpopulations (e.g. Hilker \& Richtler 2000).

In order to draw further conclusions on the origin of $\omega$ Centauri we analysed a large sample of spectra with medium resolution of stars near the age sensitive subgiant branch and main sequence turnoff region observed with FORS2/MXU at the VLT. For the metallicity calibration our dataset comprises spectra with known $[\mathrm{Fe} / \mathrm{H}]$ values: of the chemically homogeneous globular cluster M55, standard stars and a grid of synthetic spectra taken from Bailer-Jones (2000). To quantify the strength of the absorption lines we defined line indices (mostly for $\mathrm{Fe}$, but also $\mathrm{Ca}, \mathrm{Mg}$ and $\mathrm{H}$ lines) with central widths adjusted to the resolution of our spectra. As a measure for the Fe abundance we combined several line indices to a mean Fe indictor. We corrected for the temperature effect by using a temperature dependent mean Balmer index. From the derived metallicities and in combination with photometric data kindly provided by Rey et al. (2004), we were able to disentangle the age-metallicity degeneracy. We determined the ages by interpolating in a grid of Yonsei-Yale isochrones taken from Kim et al. (2002). We assumed $[\alpha / \mathrm{Fe}]=0.3$

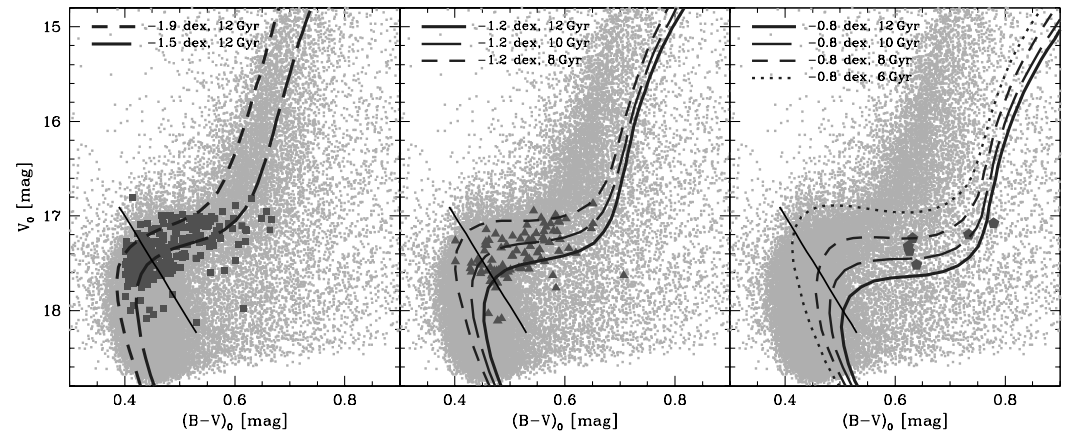

Figure 1. The position of stars of different abundances compared with isochrones of different ages and metallicities. The abundance ranges for the highlighted stars are: left: $-2.0<[\mathrm{Fe} / \mathrm{H}]<-1.4$, middle: $-1.4<[\mathrm{Fe} / \mathrm{H}]<-1.0$, right: $-1.0<[\mathrm{Fe} / \mathrm{H}]$. The dominant metal poor population is best fit by $12 \mathrm{Gyr}$ isochrones. More metal-rich stars scatter around isochrones that are up to 2 Gyr younger. The most metal-rich stars are distributed between the 9 to $11 \mathrm{Gyr}$ isochrones. 


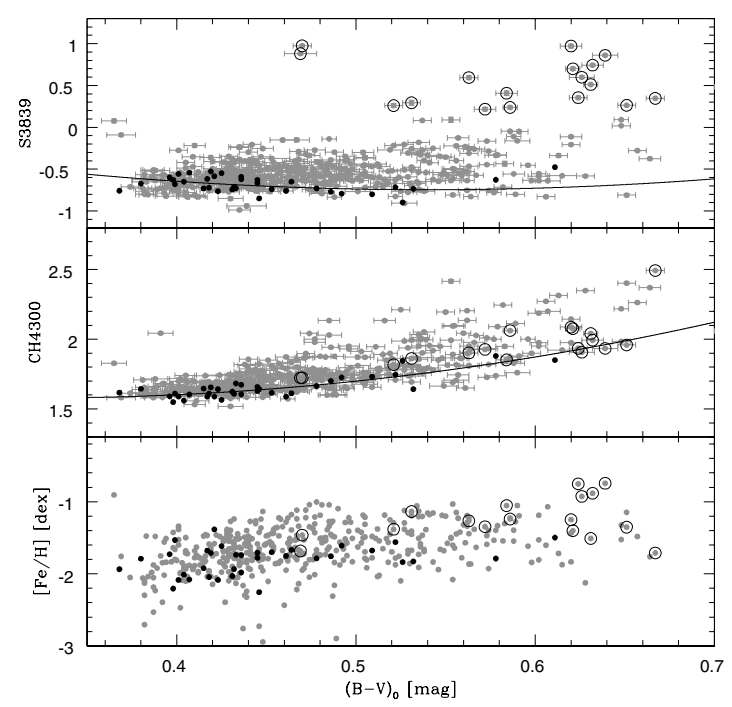

Figure 2. From top to bottom, $\mathrm{S}(3839), \mathrm{CH}(4300)$ and $[\mathrm{Fe} / \mathrm{H}]$ vs. $(B V)_{0}$ for stars in $\omega$ Cen and M55 (black). The fit curves of the M55 data indicated the temperature effect in the indices. Highlighted by circles are the most CN-rich stars. These stars are reidentified in the $\mathrm{CH}(4300)$ vs. $(B-V)_{0}$ and $[\mathrm{Fe} / \mathrm{H}]$ vs. $(B-V)_{0}$ diagrams.

dex for stars with $[\mathrm{Fe} / \mathrm{H}]<-1.0$ dex and $[\alpha / \mathrm{Fe}]=0.1$ dex for stars with $[\mathrm{Fe} / \mathrm{H}]>-1.0$ dex (Pancino et al. 2002). The resulting age-metallicity dependencies are shown in Fig. 1. Our results confirm an age spread of up to 4 Gyr in the sense that the metal-rich stars are on average younger than the dominant metal-poor population (Hilker et al. 2004).

The $\mathrm{CN}$ and $\mathrm{CH}$ absorption bands were measured by the commonly used indices $\mathrm{S}(3839)$ and $\mathrm{CH}(4300)$ (e.g. Norris et al. 1981). Again, M55 served as a comparison object for estimating the strength of the $\mathrm{CN}$ and $\mathrm{CH}$ variations and to estimate the effect of temperature on these indices. Specifically, we found that the line strength of $\mathrm{CH}(4300)$ is strongly anticorrelated with temperature, while the strength of $\mathrm{S}(3839)$ shows a very week temperature effect. The analysis of star-to-star $\mathrm{CN}$ and $\mathrm{CH}$ variations revealed, as for the RGB, the existence of a large scatter toward very high $\mathrm{CN}$ and $\mathrm{CH}$ abundances. From an inspection of the location of the most CN-rich stars in the corresponding $\mathrm{CH}(4300)$ and $[\mathrm{Fe} / \mathrm{H}]$ vs. $(B-V)_{0}$ diagrams, we found that the CN-rich stars tend to be more $\mathrm{CH}$-weak, and mainly belong to the metal-rich populations (Fig. 2).

Our study shows that $\omega$ Centauri definitely has experienced a complex chemical enrichment history with yields of different types of stars contributing. This has not been observed in any other globular cluster. The results provide further evidence for a prolonged star formation history as would be expected for a dwarf galaxy.

\section{References}

Hilker, M., Kayser, A., Richtler, T. \& Willemsen., P. 2004, A\&A 422, L9

Hilker, M. \& Richtler, T. 2000, A\&A 362, 895

Kim, Y., Demarque, P., Yi, S. K. \& Alexander, D.R. 2002, AJ 143, 499

Norris, J., Cottrell, P.L., Freeman, K.C. \& da Costa, G.S. 1981, ApJ 244, 205

Norris, J., Freeman, K.C., Mayor, M. \& Seitzer, P. 1997, ApJL 487, 187

Panino, E., Pasquini, L., Hill, V., Ferraro, F.R. \& Bellazzini, M. 2002, ApJL 568, L101

Rey, S., Lee, Y., Ree, C.H., Joo, J. \& Sohn, Y. 2004, AJ 127, 958 